\title{
Investigation of Hyperbolic Metamaterials
}

\author{
Tatjana Gric ${ }^{1,2, *}$ and Ortwin Hess ${ }^{3}$ \\ 1 Department of Electronic Systems, Vilnius Gediminas Technical University, Vilnius LT-10223, Lithuania \\ 2 Semiconductor Physics Institute, Center for Physical Sciences and Technology, Vilnius LT-02300, Lithuania \\ 3 The Blackett Laboratory, Department of Physics, Imperial College London, London SW7 2AZ, UK; \\ o.hess@imperial.ac.uk \\ * Correspondence: tatjana.gric@vgtu.lt; Tel.: +370-6167-8292
}

Received: 21 June 2018; Accepted: 12 July 2018; Published: 25 July 2018

Featured Application: It should be mentioned that the exceptional properties of SPPs, such as, subwavelength confinement and strong field enhancement give rise to a wide range of innovative applications. For example, owing to the excitation of SPPs, resolution of the so-called "superlens" has reached as high as $30 \mathrm{~nm}$. The former opens the wide avenues for optical microscopy in terms of overcoming the diffraction limit. Alternatively, the resolution can be even higher in plasmonics-assisted spectroscopy, such as surface-enhanced Raman spectroscopy (SERS). The Raman signal can be considerably amplified by SERS approach. Doing so, the vibrational information of single molecules can be probed and recorded by employing the strong local field enhancement of plasmonic composites and the chemical mechanism including charge transfer between the species and the metal surface. Tip-enhanced Raman spectroscopy (TERS) may stand for the further resolution improvement. Based on the study on TERS-based spectral imaging with extraordinary sub-molecular spatial resolution, one can definitely resolve the inner structure and surface configuration of a single molecule.

Abstract: Composites designed by employing metal/dielectric composites coupled to the components of the incident electromagnetic (EM) fields are named metamaterials (MMs), and they display features not observed in nature. This type of artificial media has attracted great interest, resulting in groundbreaking theory that bridges the gap between EM and photonic phenomena. Practical applications of MMs have been delayed due to the high losses related to the use of metallic composites, on top of the challenges in manufacturing nanoscale, three-dimensional structures. Novel materials-for instance, graphene or transparent-conducting oxides (TCOs), employed for the production of multilayered MMs - can significantly suppress undesirable losses. It is worthwhile noting that three-layered nanocomposites enable an increase in the frequency range of the surface wave. This work analyzes recent progress in the physics of multilayered MMs. We deliver an outline of key notions, such as effective medium approximation, and present multilayered MMs based on the three-layered structure. An overview of graphene multilayered MMs reveals their ability to support Ferrell-Berreman (FB) modes. We also describe the tunable properties of the multilayered MMs.

Keywords: hyperbolic metamaterial; transparent conducting oxides; graphene; surface plasmon polaritons

\section{Introduction}

At the turn of the century, a new field of research emerged that was a hybrid of physics, electrical engineering, and materials science. The study of metamaterials (MMs) addresses the rational design and arrangement of a material's building blocks to attain physical properties that may go significantly beyond those of its original constituent materials. Most often, the concept of MMs is associated with 
electromagnetic (EM) wave propagation, where the engineering of resonant subwavelength structures allows for the precise control of effective wave properties. It is this advanced functionality that enables the realization of unique wave phenomena, such as the focusing of light below the diffraction limit or EM cloaking of objects-concepts that have captured the imagination of researchers and the general public alike. Importantly, the element of functionality is an integral part of the MM concept.

Recently, propagation of EM waves on two-dimensional interfaces has stimulated tremendous interest [1,2]. Surface plasmonic polariton (SPP) [3-6] attracts lots of attention in the scientific community. SPPs have provided a fertile ground for new applications in many fields [7-14]. During last several decades, it has been demonstrated that it is possible to control SPPs in an unprecedented way by nanostructuring the metal surface. Hyperbolic MMs [15] have stimulated increasing interest because of the capability of supporting large wavenumbers. Their unusual properties opened wide avenues for many fascinating applications, such as hyperlenses for biosensing [9]. The potential biosensing applications are achievable with the novel high-frequency cut-off SPPs in hyperbolic graphene-dielectric multilayered metamaterials. For infrared frequencies, infrared plasmonic MMs were designed by $\mathrm{C}$. Wu et al. Moreover, their use has been suggested as a biosensing platform [16]. It is worthwhile to note that hyperbolic MMs have already been treated from the perspective of supporting photonic modes with unbounded k-vectors in [17]. Herein, we take a step forward by investigating various types of modes supported by multilayered structures, for example, Ferrell-Berreman (FB) and others. The former is achievable by employing the tunability properties of the graphene used as a building block of the multilayered composite. The experimental realization of a multilayered structure of alternating graphene and $\mathrm{Al}_{2} \mathrm{O}_{3}$ layers has been reported in [18]. However, the former study does not offer an overview of the tuning possibilities.

Our present discussion of multilayered MMs is divided into three parts. In the first part, fundamental physical notions that underlie graphene-dielectric multilayered MMs are discussed. Moreover, the realization of the dispersion characteristics of the SPPs, their theoretical implications, and their specific characteristics are reviewed. In addition, the concept of FB modes is introduced. In Section 4, the extension of the frequency region of the SPPs is discussed in terms of the inclusion of a third additional layer. Particular emphasis is placed on the topological structures. Finally, Section 5 discusses the possibility of the inclusion of transparent-conducting oxide (TCO) layers in multilayered MMs, specifically paying attention to the new kinds of the SPPs.

\section{Multilayered MM-Based Effective Medium Approach}

Herein, it is aimed to derive the effective medium permittivities for an anisotropic multilayered MM. The technique is based on the generalized Maxwell Garnett method [19], which seeks to derive analytical formulas describing the material in different directions, i.e. parallel $\left(\varepsilon_{\|}\right)$and perpendicular $\left(\varepsilon_{\perp}\right)$. Based on the mentioned methodology, a sufficiently dilute material is considered such that a hierarchy of macroscopic and microscopic fields can be assumed. The schematic view of a structure composed of metallic and dielectric layers making a multilayered MM is outlined in Figure 1.

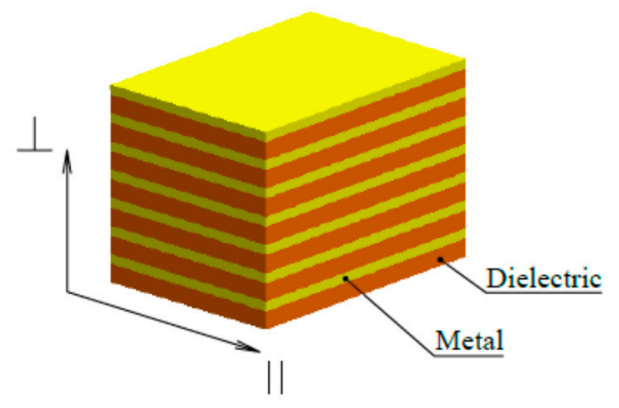

Figure 1. A view of a multilayered metamaterial (MM). 
The permittivities of the metallic and dielectric layers are $\varepsilon_{m}$ and $\varepsilon_{d}$, correspondingly. Additionally, the fill ratio of the total width of metal in the composite to the overall width of the MM can be defined as follows:

$$
\rho=\frac{d_{m}}{d_{m}+d_{d}}
$$

where $d_{m}$ is summation of the widths of metallic sheets in the composite and $d_{d}$ is summation of the widths of the dielectric sheets.

\subsection{Effective Parallel Permittivity}

Herein, an analytical formula expressing the parallel permittivity component characterizing the structure under consideration is derived. It is worthwhile mentioning that:

$$
\vec{D}=\varepsilon_{e f f} \vec{E}
$$

where $\varepsilon_{\text {eff }}$ is the effective permittivity. It is a well-known fact that the tangential component of the electric field should be continuous across a boundary of two different media. Thus, it follows:

$$
E_{m}^{\|}=E_{d}^{\|}=E^{\|}
$$

where $E_{m}^{\|}$is assigned as the electric field in the metallic layers, $E_{d}^{\|}$is the electric field in the dielectric sheets, and $E^{\|}$is the electric field of the subwavelength MM. The overall displacement might be found by averaging the displacement field components arising from the metallic and dielectric building blocks:

$$
D^{\|}=\rho D_{m}^{\|}+(1-\rho) D_{d}^{\|}
$$

Rearranging the above equation, the following equation is obtained:

$$
\varepsilon_{e f f}^{\|} E^{\|}=\rho \varepsilon_{m} E^{\|}+(1-\rho) \varepsilon_{d} E^{\|}
$$

One can conclude with the final equation, as follows:

$$
\varepsilon_{||}=\rho \varepsilon_{m}+(1-\rho) \varepsilon_{d}
$$

\subsection{Effective Perpendicular Permittivity}

It is specifically known that the normal component of the electric displacement vector at a boundary should be continuous. The former is outlined by the following expression:

$$
D_{m}^{\perp}=D_{d}^{\perp}=D^{\perp}
$$

It is also known that a superposition of the electric field components generated by the sheets dramatically affects the total magnitude of the electric field. Consequently, it can be defined by:

$$
E^{\perp}=\rho E_{m}^{\perp}+(1-\rho) E_{d}^{\perp}
$$

where $E_{m}^{\perp}$ is the perpendicular component of the electric field in the metallic area, $E_{d}^{\perp}$ is the perpendicular component of the electric field in the dielectric area, and $E^{\perp}$ is the total electric field in the MM. The boundary condition from Equation (7) and Maxwell's equation [20] can be used. Moreover, it is of particular importance to substitute them into Equation (8). If the common electric 
field components are cancelled out, and everything is solved for $\varepsilon_{\perp}$, it is possible to derive the analytic formula for the perpendicular permittivity of the multilayered MM:

$$
\varepsilon_{\perp}=\frac{\varepsilon_{m} \varepsilon_{d}}{\rho \varepsilon_{d}+(1-\rho) \varepsilon_{m}}
$$

\section{Two-Layered MMs for Surface Plasmon Polariton Guiding}

Figure 2 is a schematic view of a hyperbolic multilayered MM consisting of periodically changing graphene and dielectric layers.

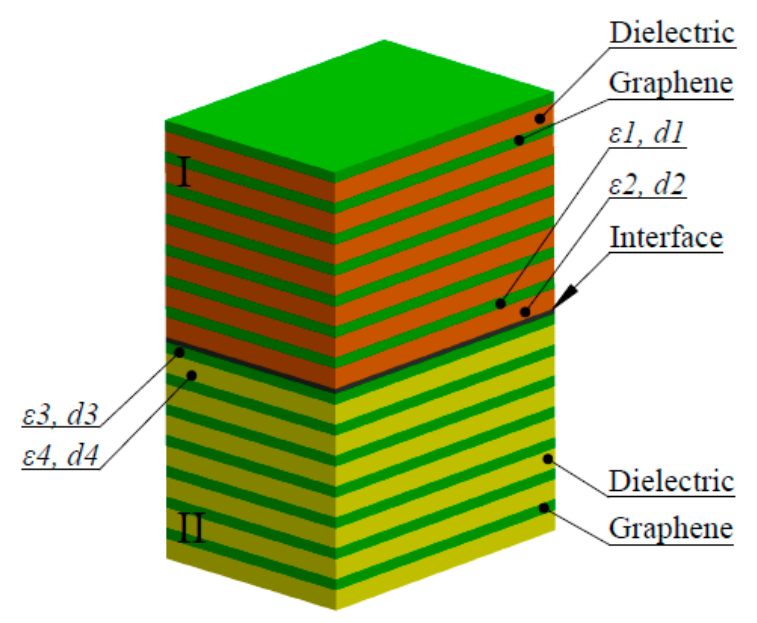

Figure 2. A schematic diagram of the boundary surface separating two different infinite multilayered MMs designed by a stack of periodically changing graphene and dielectric layers. Here, dielectric and graphene sheets are denoted by indexes "1, 2", correspondingly.

The effective-medium theory [21] can be applied if the width of any sheet is much smaller than the wavelength of radiation. This approach allows us to describe the optical response in such a structure.

Herein, we present the dispersion branches calculated after carrying out the homogenization of two MMs. The dispersion pattern for the case $\varepsilon_{1}=\varepsilon_{3}, \varepsilon_{2}=\varepsilon_{4}$ and $d_{1} \neq d_{2} \neq d_{3} \neq d_{4}$ is presented in Figure 3. Figure 3 demonstrates that the SPPs branch at the interface separating MMs with $d_{1}=0.35 \mathrm{~nm}$, $d_{2}=10 \mathrm{~nm}, d_{3}=0.25 \mathrm{~nm}$, and $d_{4}=10.1 \mathrm{~nm}$. Moreover, the dispersion diagrams of SPPs, characterized by the effective properties outlined in [21], are displayed in Figure 3.

The tunability feature of the frequency ranges of SPPs is the main outcome of changing the Fermi energy. It is worthwhile noting that the decrease of the Fermi energy $\mu$ causes movement of the dispersion diagrams to lower frequency ranges. On the contrary, the increase of the Fermi energy $\mu$ will stimulate their movement to higher frequencies. As illustrated in Figure 3, the smallest asymptotic frequency is achievable in the case of the smallest Fermi energy. The presented controllability feature provides possibilities to engineer the SPPs by the Fermi energy of the graphene layers. Consequently, special phase-matching tools, such as a grating or prism coupling [22], near-field excitation [23], and end-fire coupling [24], are needed for their excitation via three-dimensional beams [3].

Because of the graphene being treated as lossless, $\beta$ is complex resulting in a finite propagation length, presented in Figure 3b.

The next case to deal with, i.e. $\varepsilon_{1}=\varepsilon_{3}, \varepsilon_{2} \neq \varepsilon_{4}$ and $d_{1} \neq d_{2} \neq d_{3} \neq d_{4}$, is studied by sketching four modes in Figure 4 at the interface of the MMs with $\varepsilon_{4}=2.25$. As it follows from Figure 4 , the case $\mu=0.5 \mathrm{eV}$ is characterized by the smallest asymptotic frequency. It is worthwhile noting, that $\operatorname{Re}(\beta) \neq 0$ allows for the quasi-bound [21], leaky region of the dispersion equation. 

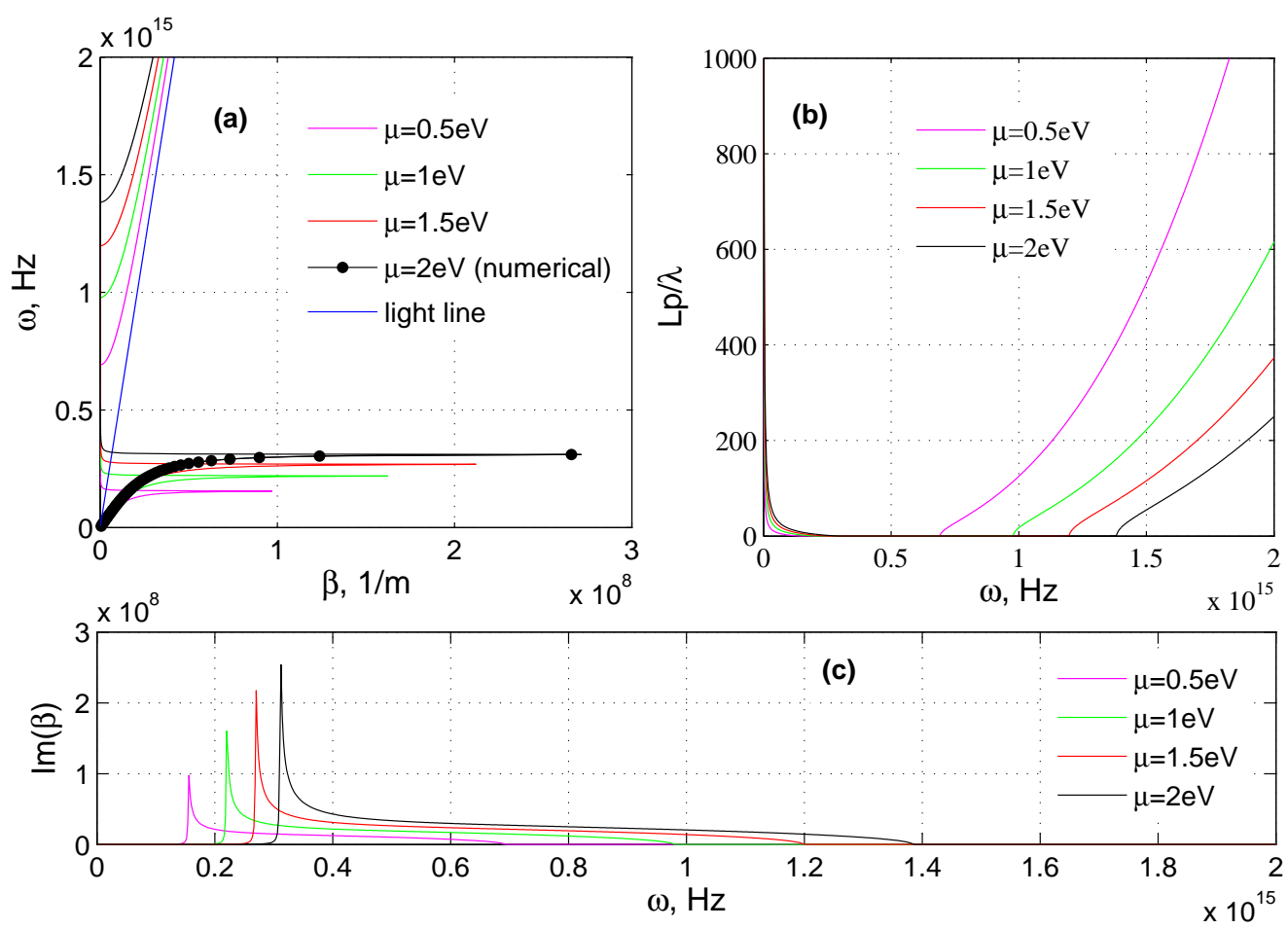

Figure 3. The dispersion of SPPs (a); propagation lengths (b) and absorption (c) at different Fermi energy, where number of graphene layers, i.e. $N=1, d_{\mathrm{d}}=10 \mathrm{~nm}, \varepsilon_{1}=\varepsilon_{3}, \varepsilon_{2}=\varepsilon_{4}$ and $d_{1} \neq d_{2} \neq d_{3} \neq d_{4}$ and the blue line in (a) denotes the free space light line.
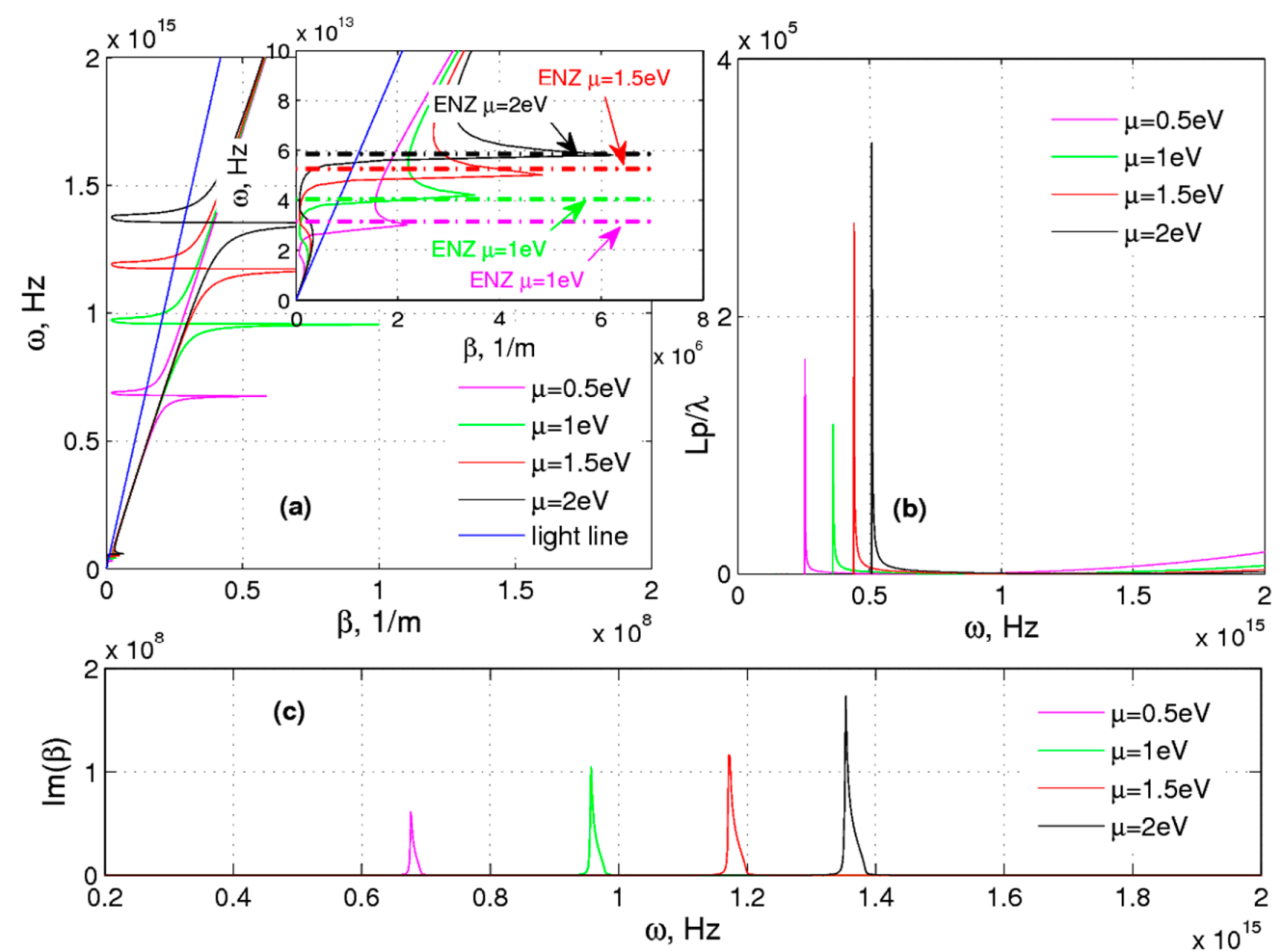

Figure 4. The dispersion of surface plasmonic polaritons (SPPs) (a); propagation lengths (b) and absorption (c) at different Fermi energies, where the number of graphene layers $N=1, d_{d}=10 \mathrm{~nm}$, $\varepsilon_{1}=\varepsilon_{3}, \varepsilon_{2} \neq \varepsilon_{4}$, and $d_{1} \neq d_{2} \neq d_{3} \neq d_{4}$, and the blue line in (a) denotes the free space light line. 
Following the previous instances, the transverse magnetic (TM) mode dispersion plots are used to deal with the case of $\varepsilon_{1}=\varepsilon_{3}, \varepsilon_{2} \neq \varepsilon_{4}$ and $d_{1}=d_{3}, d_{2}=d_{4}$. Four various modes are presented in Figure 5. The outcome of tuning the fill ratios is examined as displayed in Figure 5. Firstly, it is worth discussing how the dielectric thickness $d_{\mathrm{d}}$ impacts the dispersion branch (see Figure $5 \mathrm{a}$ ). It is found that an increase of $d_{\mathrm{d}}$ causes a shift of the upper limit to higher frequencies. Moreover, one can manipulate the frequency range for SPPs by tuning the number of graphene sheets, as shown in Figure 5c.

It should be mentioned that, following [25], it is of particular importance to omit negative values of the electrical length and absorption in Figure $3 b, c$, Figure $4 b, c$ and Figure $5 b, d-f$. The effect of absorption control was considered in [26]. It was proposed to tune and enhance the absorption of thin dye-doped polymeric films by metallic and lamellar metal-dielectric hyperbolic metamaterial substrates. Herein, by employing graphene as a building block of the hyperbolic metamaterial, we achieve a higher level of tunability by changing the Fermi energy. Additionally, the sketched graphs represent clear evidence of the existing SPPs propagating for a long distance. The former is possible because the real part of $\beta$ is very low within this spectral range. It is of particular importance to mention that the same case appears when dealing with the boundary separating the MM and isotropic medium [27].
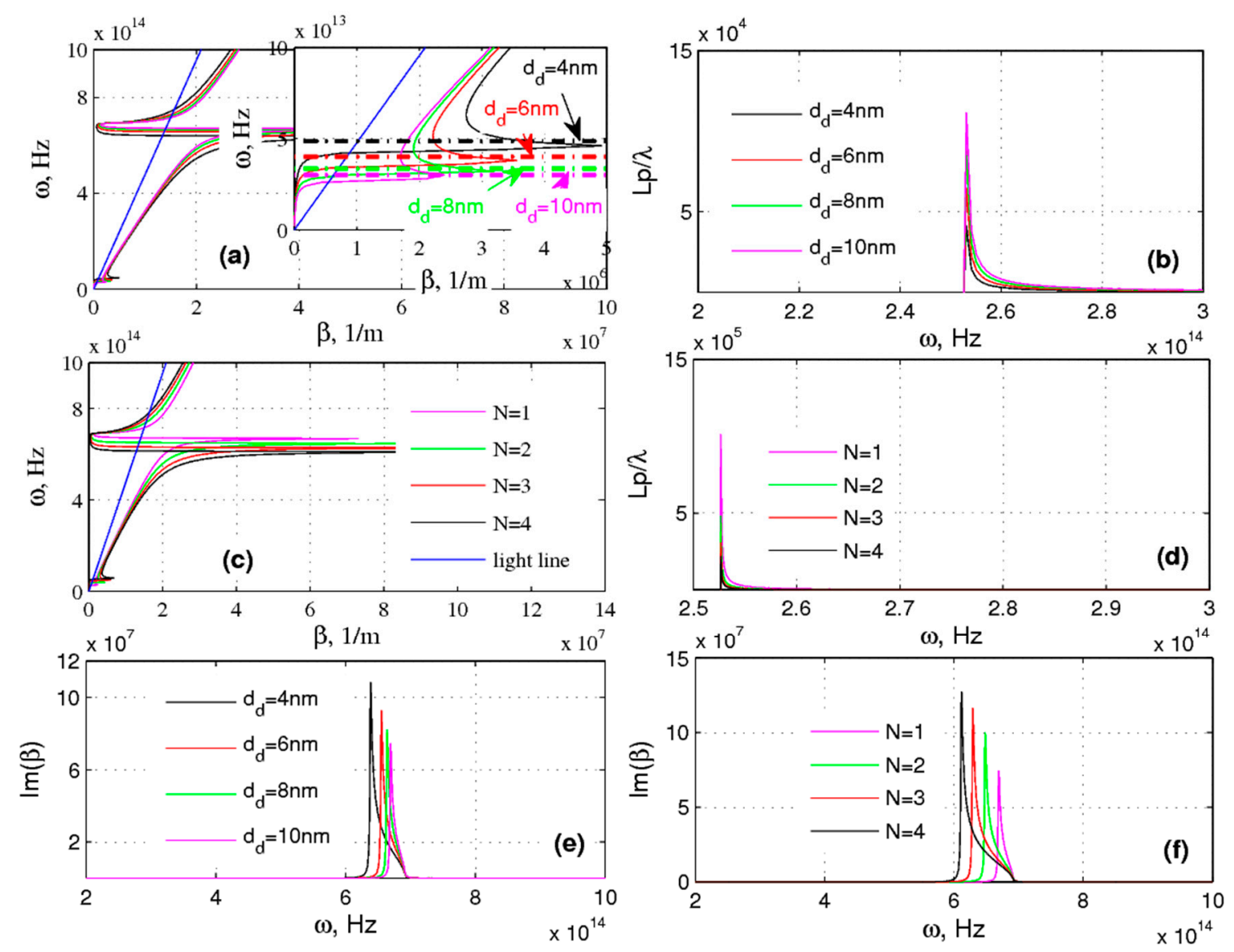

Figure 5. The dependences of dispersion of SPPs, propagation lengths, and absorption on $(\mathbf{a}, \mathbf{b}, \mathbf{e})$ the thickness of dielectric $d_{\mathrm{d}}$ and (c,d,f) number of graphene layers. Herein, the number of graphene layers, i.e., $N=1, \mu=0.5 \mathrm{eV}$ in $(\mathbf{a}, \mathbf{b}, \mathbf{e})$ and $d_{\mathrm{d}}=10 \mathrm{~nm}, \mu=0.5 \mathrm{eV}$ in $(\mathbf{c}, \mathbf{d}, \mathbf{f})$.

The imaginary parts of the wavevector (i.e., absorption) are demonstrated in Figures $3 c, 4 c$ and 5 e,f. The absorption resonances allow for the rich phenomenon, i.e., behavior of the simple 
multilayered MMs as directive and monochromatic thermal sources [28] without possessing any periodic corrugation.

We used a modal analysis to demonstrate that the bulk absorption in MMs arises because of the excitation of leaky bulk polaritons named FB modes [29-31]. It is worthwhile noting that the interaction of the bulk plasmon with free space light at frequencies close to the metal epsilon-near-zero (ENZ) [32] is possible. This method has been addressed by Ferrell for metallic foils in [33], and by Berreman for polar dielectric films in [34]. Radiative excitations called FB modes are possible due to the application of the multilayered MMs with graphene layers. It should be noted that these FB modes differ from SPPs, possibly due to the usage of metal foils. The former occurs because of energy propagating along the surfaces of the metal in cases of SPP modes. With respect to Figures 3a and $4 a, c$, particular attention should be paid to FB modes existing at energies close to the ENZ of the hyperbolic MM to the left of the light line. A specific example of a three-layered MM and its dispersion engineering, allowing the development of new devices, are studied in detail in the next section.

\section{Three-Layered MMs for Surface Plasmon Polariton Guiding}

Figure 6 illustrates a diagram of dielectric/graphene/dielectric multilayered MM.

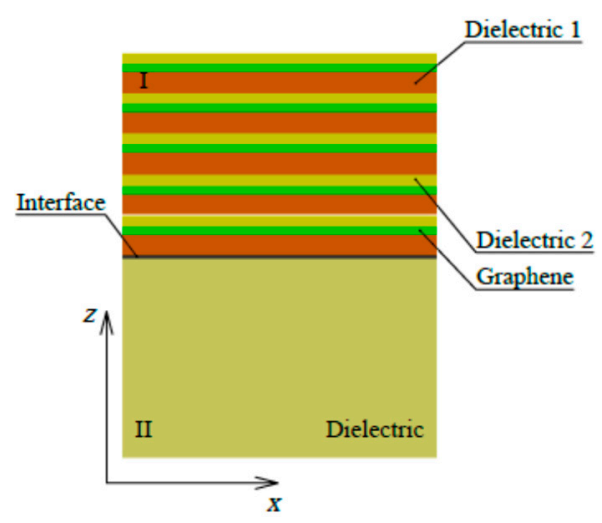

Figure 6. Schematic of graphene-dielectric multilayered MM.

It should be mentioned that the MM under study is described by effective permittivity tensor as follows:

$$
\varepsilon=\varepsilon_{0}\left(\begin{array}{ccc}
\varepsilon_{\|} & 0 & 0 \\
0 & \varepsilon_{\|} & 0 \\
0 & 0 & \varepsilon_{\perp}
\end{array}\right)
$$

in the principal-axis coordinate system. The principal values of the tensor are obtained in $[35,36]$ by means of the theoretical approach provided in Section 2:

$$
\begin{gathered}
\varepsilon_{\|}=f_{g} \varepsilon_{g}+f_{1} \varepsilon_{1}+\left(1-f_{g}-f_{1}\right) \varepsilon_{2} \\
\varepsilon_{\perp}=\varepsilon_{g} \varepsilon_{1} \varepsilon_{2} /\left(f_{g} \varepsilon_{1} \varepsilon_{2}+f_{1} \varepsilon_{g} \varepsilon_{2}+\left(1-f_{g}-f_{1}\right) \varepsilon_{g} \varepsilon_{1}\right)
\end{gathered}
$$

where $f_{g}=d_{g} / L$ and $f_{1}=d_{1} / L, L=d_{1}+d_{2}+d_{g}$ represent the graphene and first dielectric filling ratios, respectively, $d_{g}$ is the thickness of the graphene layer, $d_{1}$ is the thickness of the first dielectric sheet, $d_{2}$ is the thickness of the second dielectric layer, $\varepsilon_{1}$ is the permittivity of the first dielectric, and $\varepsilon_{2}$ is the permittivity of the second dielectric.

For the different cases presented in Figure 7, dispersion relations are obtained as follows

$$
\beta=k \sqrt{\frac{\varepsilon_{1} \varepsilon_{2} \varepsilon_{g}\left(\varepsilon_{1}-\varepsilon_{2}-\varepsilon_{1} f_{1}+\varepsilon_{2} f_{1}+\varepsilon_{2} f_{g}-\varepsilon_{g} f_{g}\right)}{\varepsilon_{1}^{2} \varepsilon_{g}-\varepsilon_{2}^{2} \varepsilon_{g}-\varepsilon_{1}^{2} \varepsilon_{g} f_{1}+\varepsilon_{2}^{2} \varepsilon_{g} f_{1}+\varepsilon_{1}^{2} \varepsilon_{2} f_{g}-\varepsilon_{1}^{2} \varepsilon_{g} f_{g}-\varepsilon_{2} \varepsilon_{g}^{2} f_{g}+\varepsilon_{2}^{2} \varepsilon_{g} f_{g}}}
$$




$$
\begin{gathered}
\beta=k \sqrt{\frac{\varepsilon_{1} \varepsilon_{2} \varepsilon_{g}\left(\varepsilon_{1} f_{1}-\varepsilon_{2} f_{1}-\varepsilon_{2} f_{g}+\varepsilon_{g} f_{g}\right)}{f_{1} \varepsilon_{1}^{2} \varepsilon_{g}-f_{g} \varepsilon_{1} \varepsilon_{2}^{2}+f_{g} \varepsilon_{1} \varepsilon_{g}^{2}-f_{1} \varepsilon_{2}^{2} \varepsilon_{g}}} \\
\beta=k \sqrt{\frac{\varepsilon_{1} \varepsilon_{g}}{\varepsilon_{1}+\varepsilon_{g}}} \\
\beta=k \sqrt{\frac{\varepsilon_{2} \varepsilon_{g}}{\varepsilon_{2}+\varepsilon_{g}}}
\end{gathered}
$$
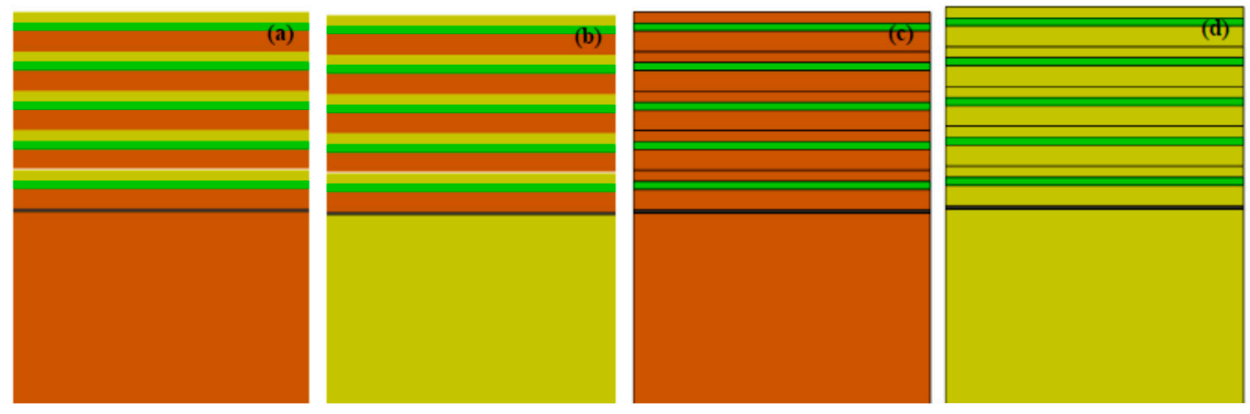

Figure 7. Schematic of graphene-dielectric multilayered MM. The figures correspond to (a) Equation (13), (b) Equation (14), (c) Equation (15), (d) Equation (16).

In consequence, a surprising result is obtained: the thicknesses of the layers (Figure 7c,d) do not affect the dispersion of a (single) boundary mode, and it agrees with the dispersion of a conventional SPP at a metal-dielectric boundary. It can be concluded that the systems displayed in Figure 7c,d can support topological properties.

Herein, we present dispersion diagrams calculated after carrying out the homogenization of two MMs. Thus, Figure 8 shows the dispersion branches of different cases of the multilayered MM. The dispersion branches of spoof SPPs at the MM interface with $d_{\mathrm{g}}=0.35 \mathrm{~nm}$ are shown in Figure 8 . It should be noted that the case displayed in Figure $8 \mathrm{a}\left(\varepsilon_{1}=18.8\right)$ corresponds to Equation (15). Thus, it can be concluded that the usage of the three-layered MM allows for the rich phenomenon, i.e., broadening of the frequency range of the SPP's existence by approximately $800 \mathrm{THz}$. The dispersion curve computed for the three-layered MM case has special features deserving of attention, besides the fact that the graph resembles behavior of a traditional metal SPP and multilayered MMs [21].

For SPPs supported by the multilayered MM under study, the horizontal asymptote appears when $\beta$ approaches infinity. Based on the dispersion equation [21], the corresponding condition is as follows:

$$
\varepsilon_{\|}^{L} \varepsilon_{\perp}^{L}-\left(\varepsilon^{R}\right)^{2}=0
$$

In other words, the position of horizontal asymptotes is determined by Equation (17). It is also concluded that $\varepsilon_{\| \mid}^{L}$ must share the same sign with $\varepsilon_{\perp}^{L}$. It is worthwhile noting that $\varepsilon^{R}$ will drastically affect the curve type.

Equation (17) holds only when both $\varepsilon_{\|}^{L}$ and $\varepsilon_{\perp}^{L}$ are negative when $\left(\varepsilon^{R}\right)^{2}$ is larger than $\lim _{\omega \rightarrow \infty} \varepsilon_{\|} \varepsilon_{\perp}$. The former scenario matches band 2 in Figure 9. Consequently, the dispersion branch possesses only one horizontal asymptote resembling the metal SPP case. The dispersion branch under such constraints bears a close resemblance to a metal and multilayered MM SPP [21] (see Figure 8).

Similar to $\omega_{\text {sp }}$ for metal, the upper bound for SPP is marked by the location of the lowest horizontal asymptote. $\omega_{\text {upper }}$ denotes the upper bound frequency, aiming to separate the upper bound of multilayered SPPs and that of metal SPPs (i.e., $\omega_{\text {sp }}$ ). One can easily prove that it is possible to excite multilayered SPPs at any frequency lower than $\omega_{\text {upper }}$ in band 2. 
The dispersion curve (Figure 8) is obtained following the hypothesis of the EM field decaying exponentially in the $z$ direction. It should be noted that not all modes with a certain frequency below $\omega_{\text {upper }}$ is SPP in this study. The former takes place even though the dispersion curve displayed in Figure 10 resembles the behavior of conventional metal SPP. One should be assured that both $k_{z}^{L}$ and $k_{z}^{R}$ are imaginary when aiming to obtain the multilayered SPPs. It can be concluded, based on Figure 10, that the two cut-off points, $\omega_{\text {lower }}$ and $\omega_{\text {upper }}$ cut the frequency range into three regions. One may define $\omega_{\text {upper }}$ as the frequency of the lowest horizontal asymptote in the dispersion branch. It is worthwhile noting that $\omega_{\text {lower }}$, which is lower than $\omega_{\text {upper, }}$, is the frequency where $k_{z}^{L}$ and $k_{z}^{R}$ are converted from real to imaginary.

The imaginary part of $k_{z}^{L}$ in a multilayered MM is negligible, while the real part exists as indicated in Figure 10, in region 1. The former proves the possibilities of the EM wave propagating into the material, resulting in a little damping. Region 1 is ended by frequency $\omega_{\text {lower }}$. The same frequency begins region $2 . k_{z}^{L}$ possesses a negligible real part and relatively large imaginary part in region 2 . The former describes the evanescence of the field is in this region. The imaginary part of $k_{z}^{L}$ disappears and the real part of $k_{z}^{L}$ occurs if $\omega_{\text {upper }}$ is lower than the frequency (in region 3 ). The wavevector component $k_{z}^{R}$ in the dielectric is shown in the right figure in Figure 10. In the same way, the wave can propagate into the dielectric in areas 1 and 3, however, it is bounded to the boundary in area 2.

The SPPs can be controlled by tuning the Fermi energy. The former is in line with the dependence of $\varepsilon_{\perp}$ on the Fermi energy [21]. It is worthwhile noting that the decreases in the Fermi energy $\mu$ will cause a shift of the dispersion curves to higher wavelengths or lower frequencies. On the contrary, they will be shifted to higher frequencies because of the increases in the Fermi energy $\mu$. The smallest asymptotic frequency is attained in the case of the smallest Fermi energy, as indicated in Figure 9. The former tunability property enables the SPP to be modified by the Fermi energy of the graphene layers.
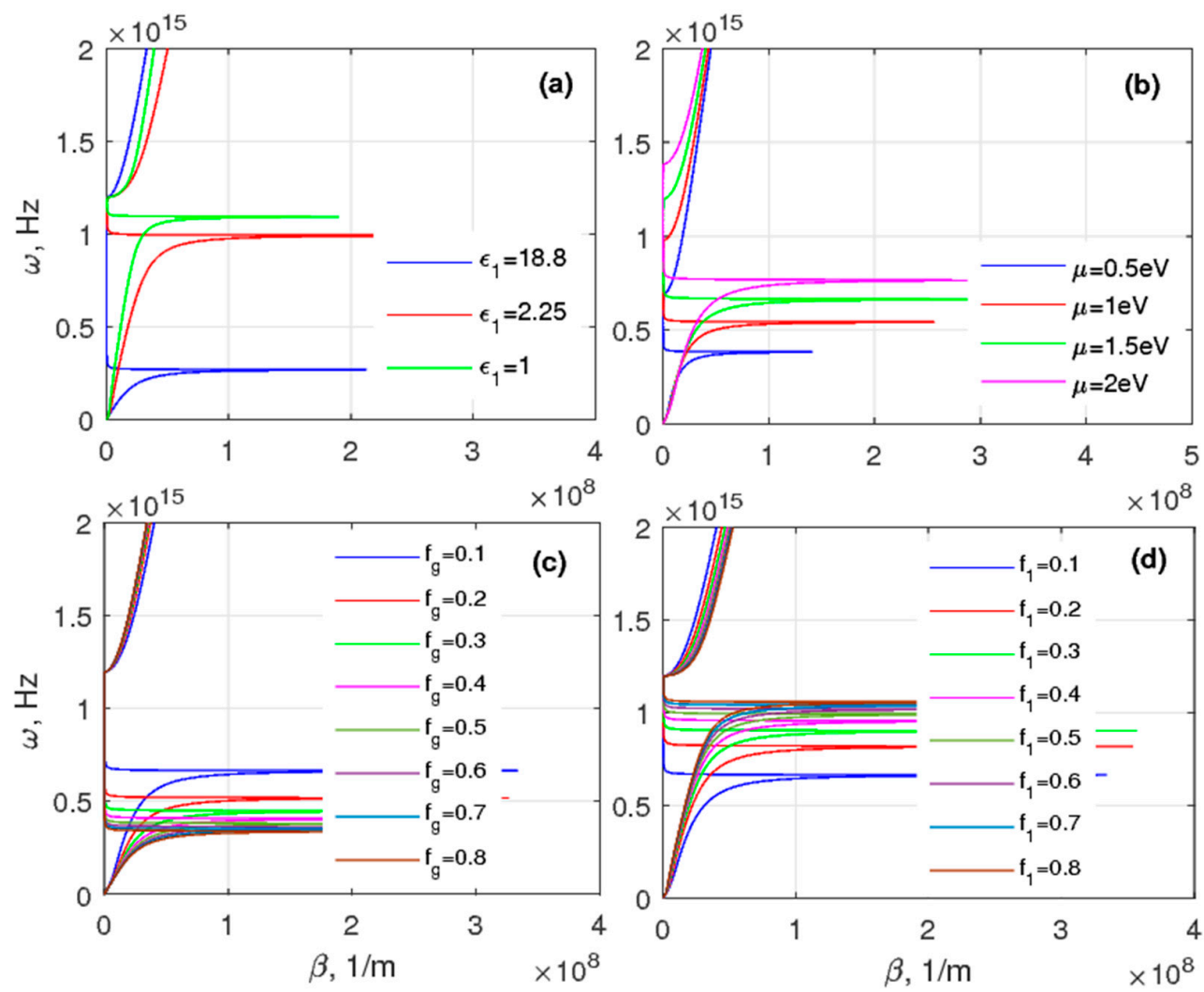

Figure 8. The impact of (a) $\varepsilon_{1}$, (b) Fermi energy $\mu$, (c) graphene filling ratio, $f_{\mathrm{g}}$, and (d) dielectric filling ratio $f_{1}$ on the dispersion diagrams of the SPPs. $f_{\mathrm{g}}=0.1, f_{\mathrm{a}}=0.5$ in $(\mathbf{a}) \cdot f_{\mathrm{g}}=0.1, f_{\mathrm{a}}=0.1$ in $(\mathbf{b}) \cdot \mu=1.5 \mathrm{eV}$ in $(\mathbf{a}, \mathbf{c}, \mathbf{d})$. 
Considering the next case, i.e., $\varepsilon_{1} \neq$ const, in Figure $8 a$, three modes are represented at the interface of MM with $\varepsilon_{2}=18.8$. As displayed in Figure $8 \mathrm{a}$, the smallest asymptotic frequency matches the case $\varepsilon_{1}=18.8$. Also, $\operatorname{Re}(\beta) \neq 0$ allows for the presence of the quasi-bound [37], leaky range of the dispersion equation. Consequently, $\beta$ does not approach infinity as the surface plasmon frequency is attained.

The dispersion curves of the TM modes are computed while aiming to address the case $f_{\mathrm{g}} \neq$ const and $f_{1} \neq$ const. In Figure $8 \mathrm{c}, \mathrm{d}$, eight different branches are presented. One should consider the impact of the fill ratios of the dielectric and graphene layer, as sketched in Figure 8c,d. First, the impact of the width of the graphene $f_{\mathrm{g}}$ on the dispersion branch (see Figure $8 \mathrm{c}$ ) should be discussed. It is found that the decrease of $f_{\mathrm{g}}$ causes a shift of the upper limit to higher frequencies. Additionally, one can control the frequency range of SPPs by changing the dielectric filling ratio, as demonstrated in Figure 8d.
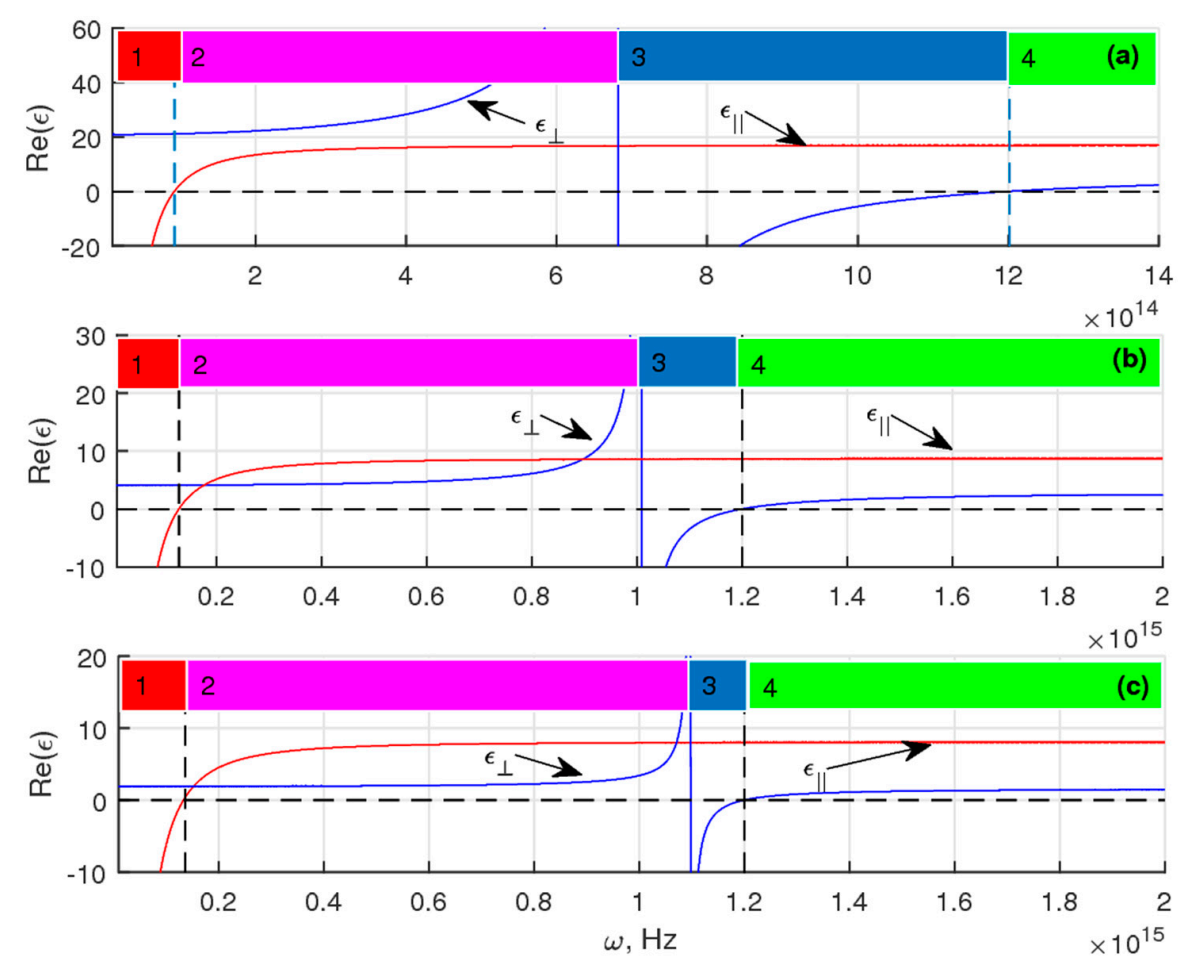

Figure 9. Real parts of $\varepsilon_{\perp}$ and $\varepsilon_{\|}$as a function of frequency. $\varepsilon_{1}=18.8$ in (a), $\varepsilon_{1}=2.25$ in (b), $\varepsilon_{1}=1$ in (c).
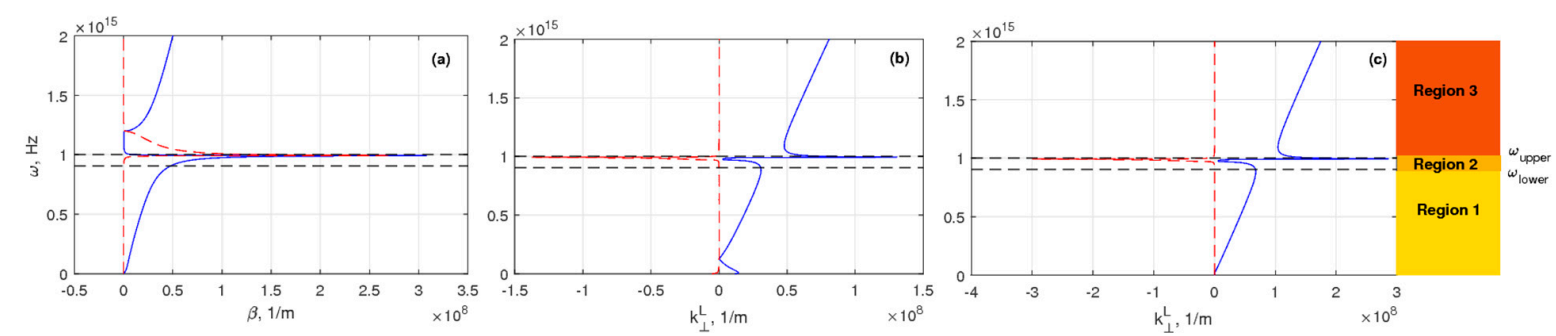

Figure 10. Each component of wavevector, when $\varepsilon_{\mathrm{R}}=18.8$. The components of $\beta$ (a) and $k$ in both multilayered MMs (b) and free space (c).

We will address the tunable hyperbolic MM made of TCO-dielectric multilayered MMs in the next section, aiming to obtain a new type of SPP. 


\section{Tunable Hyperbolic MM Made of TCO-Dielectric Multilayered MMs}

The proposed MM is displayed in Figure 11. Herein, $d_{T C O}$ and $d_{d}$ are the widths of the TCO and dielectric sheets, correspondingly. A TCO/PbS MM is considered, aiming to study the dispersive properties of SPPs. It is assumed that $\varepsilon_{d}=18.8$ for PbS sheets, and $\varepsilon_{T C O}$ is computed by means of the parameters outlined in [38].

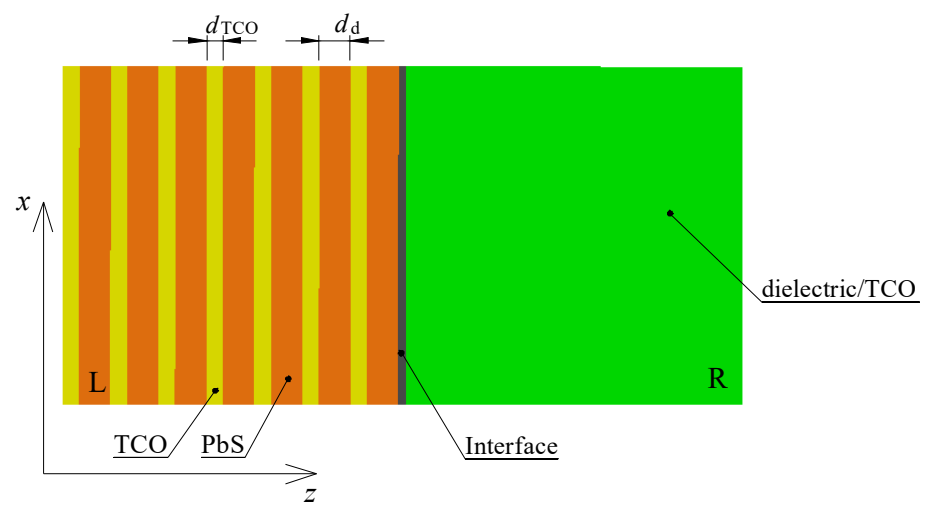

Figure 11. Interface of multilayered $M M$ made of periodically changing transparent-conducting oxide (TCO) and dielectric layers. The MM/dielectric $\left(\varepsilon_{R}=1\right.$ or $\left.\varepsilon_{R}=2.25\right)$ and MM/TCO boundaries are investigated.

For the instance of the anisotropic material, the EM wave dispersions can be sketched for $\mathrm{MM}$ /dielectric and the MM/TCO instances. Let us concentrate on a semi-infinite AZO (Aluminum Zinc Oxide)/PbS MM example while tackling the numerical calculations. Moreover, a homogenization of the MM matching the MM/dielectric and MM/TCO boundaries is considered. Sketching the branches of the permittivities $\left(\varepsilon_{\perp}\right.$ and $\left.\varepsilon_{\|}\right)$and the dielectric constant $\left(\varepsilon_{R}\right)$ allows us to consider the optical properties. Moreover, we consider different frequency ranges, distinguishing them based on the properties of and relation among $\varepsilon_{\perp}, \varepsilon_{\|}$, and $\varepsilon_{R}[39]$.

(1) $\varepsilon_{\perp}>\varepsilon_{R}>\varepsilon_{\| \mid}$and $\varepsilon_{\|}<0$ (cyan);

(2) $\varepsilon_{\perp}>\varepsilon_{R}>\varepsilon_{\| \mid}$(green);

(3) $\varepsilon_{\|}>\varepsilon_{R}>\varepsilon_{\perp}$ and $\varepsilon_{\perp}<0$ (gray);

(4) $\varepsilon_{R}>\varepsilon_{\|}>\varepsilon_{\perp}$ and $\varepsilon_{\perp}<0$ (magenta) and

(5) $\varepsilon_{\perp}<\varepsilon_{\|}<0$ (orange).

It is worthwhile mentioning that the effective parameters will dramatically influence the SPPs, as outlined in [39]. The TCO filling ratio, $f_{\mathrm{TCO}}$, is used here as the main mechanism controlling the effective optical parameters. Figures 12 and 13 display characteristic dispersion branches, considering different TCO filling factors. Different branch colors are applied, aiming to distinguish various frequency ranges. One can distinguish three SPPs fitting three different ranges in the case of $f_{T C O}=0.3$ and $\mathrm{MM} /$ dielectric boundary. Moreover, two kinds of SPPs are observed in the instance of the $\mathrm{MM} / \mathrm{TCO}$ boundary. For the MM/TCO case, the upper waves are in the gray region. The $f_{T C O}=0.5$, $0.7,0.9$ cases are associated with three exceptional cases. In the case of $f_{\mathrm{TCO}}=0.5$, two types of SPPs appear in two color ranges, i.e., cyan and gray for the MM/dielectric and MM/TCO boundaries. The upper short branches in the case of $f_{T C O}=0.7$, lying in the gray range, are presented in Figures 12 and 13. The other SPPs belong to the orange range. The supplementary SSPs belonging to the magenta range are observed with respect to a $\mathrm{MM} /$ dielectric boundary. A dark-magenta color displays an extension approach for the magenta region in the case of $\varepsilon_{R}=2.25$. One may observe two kinds of SPPs belonging to the orange and cyan ranges for the MM/TCO instance and three types of SPPs lying in the cyan, orange, and gray ranges for the $\mathrm{MM} /$ dielectric case with a filling factor $f_{T C O}=0.9$. 
It should be underlined that one SPP occurs in the cyan range for different TCO filling factors in the $\mathrm{MM} /$ dielectric case. The former is outlined in Figure 12. It is of importance that the case $\varepsilon_{R}=\varepsilon_{\text {ITO }}$ opens the gateway for possible applications, as the SPP always occurs in the cyan range for different filling factors (Figure 13).

Following the essential condition for the presence of the Dyakonov-like SPP (DSW) [35], the SPP in the green resembles the behavior of a DSW. Thus, it is important to address DSW [40], or the Dyakonov defined in [35]. Furthermore, the frequency region of the DSW can be extended by using the $\varepsilon_{R}=2.25$ material at the right-hand side of the boundary (Figure 12). The dark-green color [39] is applied, seeking to outline the former extension. Particularly, the extension of the gray region [39] is probable because of the usage of ITO (Indium Tin Oxide) instead of GZO ( $\mathrm{ZnO}: \mathrm{Ga}$ ) at the right-hand side of the boundary [39]. The SPP in the orange range is similar to the traditional SPP. The former happens because of the negative principal values of the effective permittivity in the orange range. Thus, this SPP should be associated with an SPP of a traditional type. The others are novel kinds of SPPs. Therefore, all the SPPs belong to the five different types, located in the five color ranges in Figure 12, correspondingly. In the instance of the AZO/PbS MM and air/dielectric boundary, five types of SPPs appear, and only three are found for the AZO/PbS MM and TCO interface.
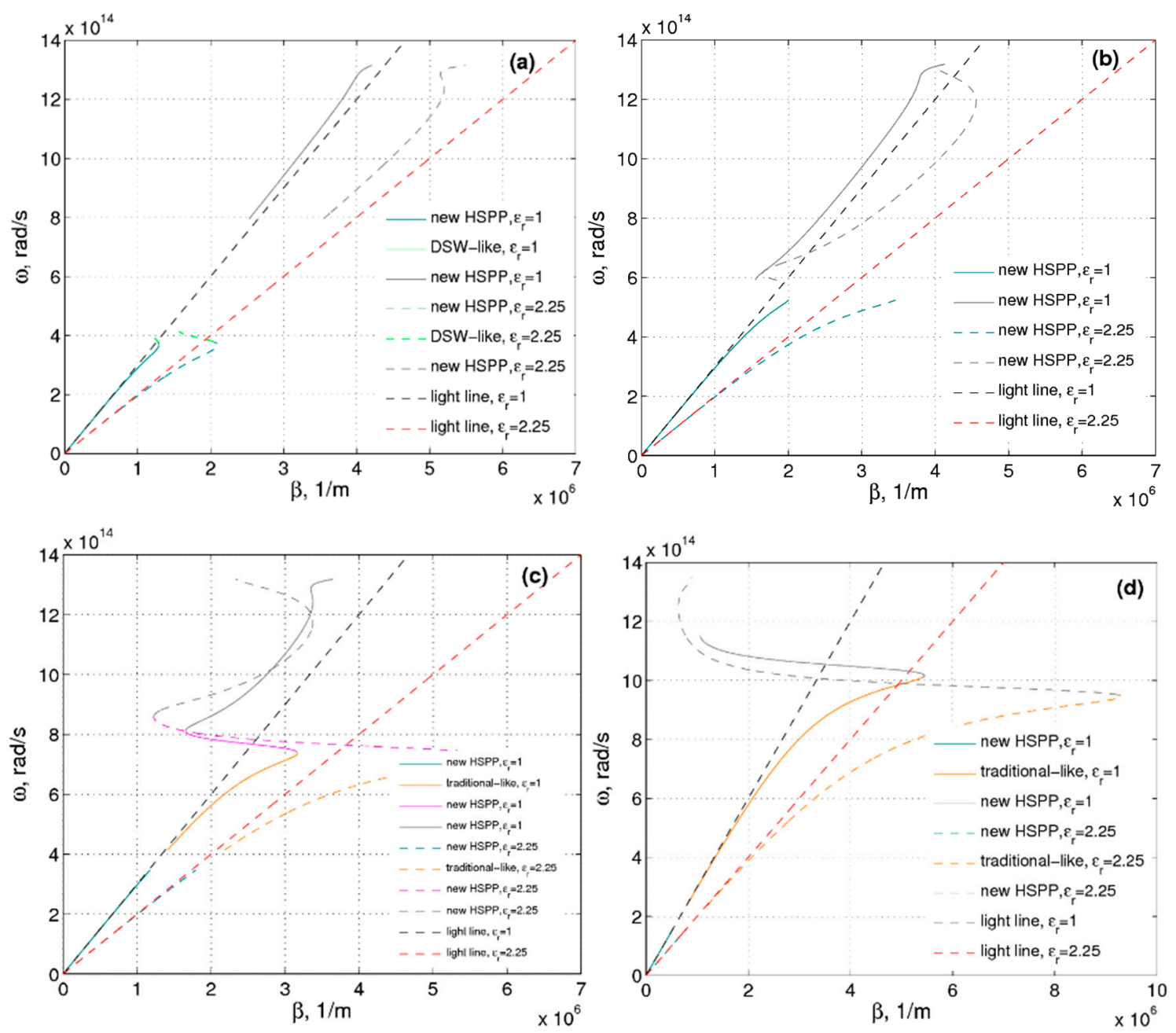

Figure 12. SPP dispersion for different TCO filling factors, i.e., $f_{T C O}=0.3$ in (a), $f_{T C O}=0.5$ in (b), $f_{T C O}=0.7$ in $(\mathbf{c})$, and $f_{T C O}=0.9$ in (d), matching the MM/dielectric boundary.

Unavoidably, a traditional-like SPP is characterized by an ordinary dispersion for the $\mathrm{MM} /$ dielectric boundary, occurring to the right of the light line. Altogether, the degradation of the dispersion in the orange range might be observed in the instance of the MM/TCO boundary. One 
can manipulate the dispersion features controlling the TCO filling factor in the MM realization. It should be noted that for a low TCO filling factor, in the case of the MM/TCO boundary, two kinds of the modes always exist, while for higher TCO filling factors, modes in the gray range disappear (Figure 13d).
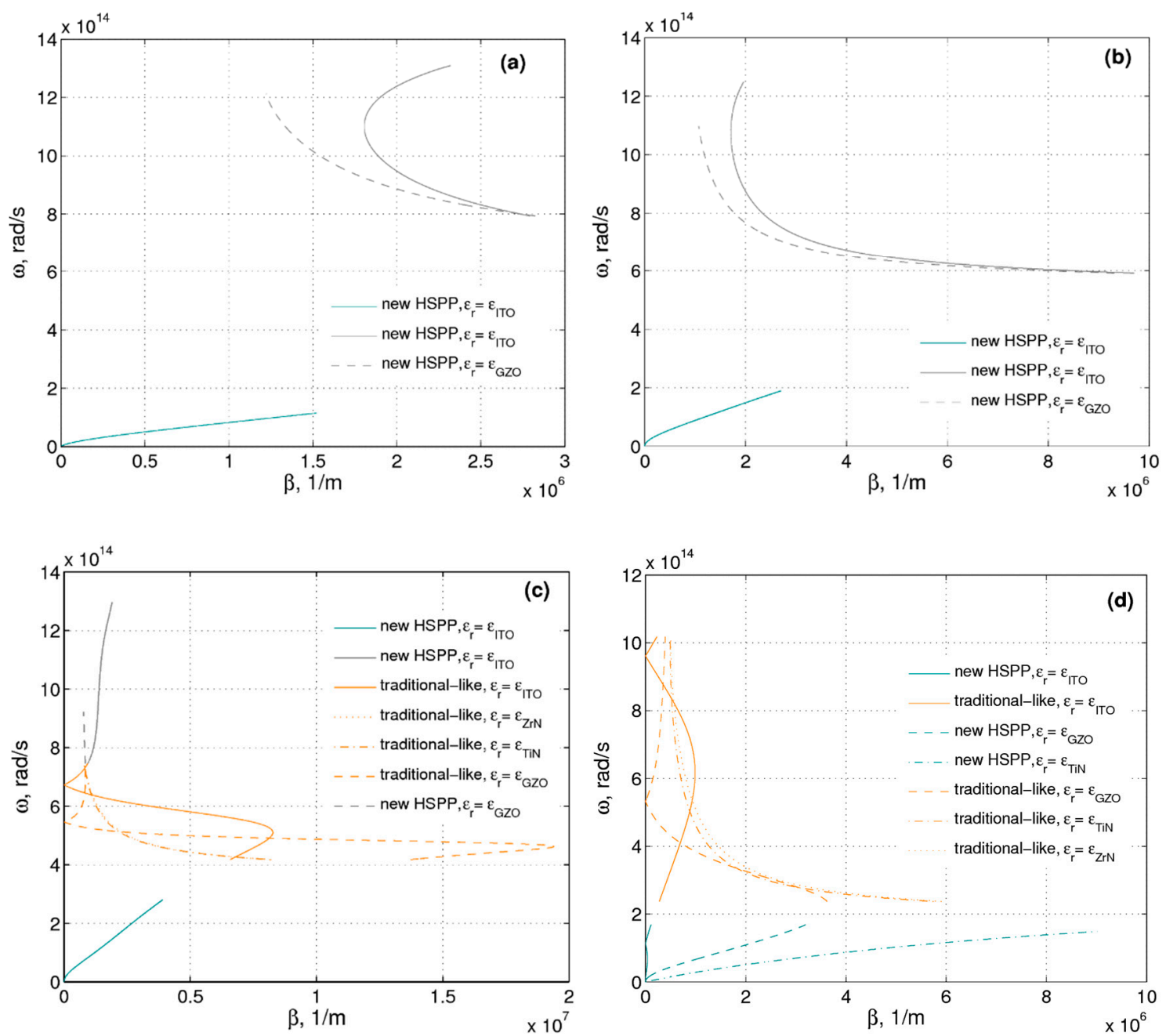

Figure 13. SPP dispersion for different TCO filling factors, i.e., $f_{T C O}=0.3$ in (a), $f_{T C O}=0.5$ in (b), $f_{T C O}=0.7$ in $(\mathbf{c})$, and $f_{T C O}=0.9$ in $(\mathbf{d})$, matching the MM/TCO boundary.

It should be mentioned that the complex mode diagrams (Figures 12 and 13), which match either the MM/dielectric or MM/TCO boundary, appear due to the confinement of SPPs in the direction perpendicular to the wave propagation. The origin of these EM SPPs is caused by the coupling of the EM fields to the oscillations of the conductor's electron plasma.

\section{Conclusions}

\subsection{Two-Layered MMs for Surface Plasmon Polariton Guiding}

To conclude, we have presented a research approach to describe the dispersion of hyperbolic MMs. The aim of these studies is to mitigate the problem under consideration, offering tuning possibilities of the hyperbolic features. The former is tremendously needed from the perspective of device applications. In this regard, we presented a multilayered MM. In the light of the present work, we have outlined the tunable features of the effective permittivity of the graphene-based MMs. Three significant outcomes should be outlined, in view of the obtained results. Firstly, compared with the 
dispersion equation in the instance of $\varepsilon_{1}=\varepsilon_{3}, \varepsilon_{2}=\varepsilon_{4}$, and $d_{1} \neq d_{2} \neq d_{3} \neq d_{4}$, the quasibound, leaky part of the dispersion equation is narrowed for the case $\varepsilon_{1}=\varepsilon_{3}, \varepsilon_{2} \neq \varepsilon_{4}$ and $d_{1} \neq d_{2} \neq d_{3} \neq d_{4}$. Secondly, one may control the perpendicular component of effective permittivity by manipulating the Fermi energy, the width of dielectric, and the number of graphene sheets in the MMs. Thirdly, one can engineer the frequency range of the SPP by changing the Fermi energy of graphene and the fill ratio of the dielectric or graphene layers in the unit cell of the MMs.

\subsection{Tunable Hyperbolic MM Made of TCO-Dielectric Multilayered MMs}

SPPs in a TCO-dielectric MM have been studied. Like graphene-dielectric MM [21], a TCO-dielectric MM support traditional-like SPPs possessing various diagrams matching two various boundaries. Based on theoretical considerations [40], the dispersion equations of SPPs were obtained. Five types of SPPs, among which three types are novel SPP types, one is DSW, and another is the traditional-like SPP, are foreseen. The existence of these SPPs is impacted by the features of the principal values of the effective permittivity. It should be noted that the presented (new) types of SPPs appear due to the principal values of the effective pemittivity being functions of frequency. Additionally, SPPs with a dispersion coinciding with the dispersion of a surface plasmon at the interface of two isotropic media are foreseen to match the MM boundary if the material at the right-hand side is the same as that used in the MM.

\subsection{Three-Layered MMs for Surface Plasmon Polariton Guiding}

SPP supported by graphene/dielectric multilayered MMs was studied theoretically. It is concluded that SPP can be supported by a three-layered MM. Also, one can easily manipulate the characteristics of the multilayered SPPs by manipulating the structural parameters. Thus, the active materials can be included into the MM design, which might open wide avenues for future devices.

The dielectric permittivity should be smaller than the absolute value of $\operatorname{Re}\left(\varepsilon_{m}\right)$, aiming to fulfill the EM boundary condition and statements of continuity [41] in the case of a traditional metal SPP. If not, the SPP mode would be cut off. The property of anisotropic material is represented by multilayered MMs if the case is considered from the effective medium perspective. Further, it is shown in Figure 9 that one may deal with multilayered MMs as with anisotropic metal near the upper bound of its SPP frequency region (in band 2). The $\operatorname{Re}\left(\varepsilon_{\perp}\right)$ is far from zero, while $\operatorname{Re}\left(\varepsilon_{\|}\right)$is much closer to zero within band 2 of Figure 9, compared with $\operatorname{Re}\left(\varepsilon_{m}\right)$ of pure metal.

The effective permittivity is caused by the interaction of the EM field with the free electrons in graphene sheets and the SPP mode coupling and resonance in multilayered MM. Consequently, the SPP frequency region is extended due to the SPP mode coupling and resonance in the multilayered MMs, characterized by anisotropic permittivity.

It is worthwhile noting that the multilayered SPPs could also be excited by means of the evanescent EM field generated by a prism or waveguide. Extended frequency range, lower propagation loss, and controllable features are possible due to the changes of the graphene/dielectric ratio, and these properties might open a gateway for possible applications in biosensor and active SPP devices.

Herein, we have theoretically considered the SPP guided by dielectric/graphene/dielectric multilayered MMs. One may conclude that an SPP can propagate when the MM is at the frequency where a conventional metal SPP cannot occur, as in the case of high permittivity of dielectric on metal. In addition to the variance of a high frequency cut-off point in comparison with metal SPPs, it was observed that a low cut-off frequency, below which SPP could not be supported by the MM, exists.

These outstanding properties open wide avenues for the usage of an SPP mode for some potential applications, which are impossible for metal SPPs. In addition to the characteristics of the multilayered SPPs being easily controlled by tuning the composite parameters, the active materials can also be incorporated into the MM. This described feature might also create new possibilities for future devices.

Funding: This research received no external funding. 
Conflicts of Interest: The authors declare no conflict of interest.

\section{References}

1. Zapata-Rodriguez, C.J.; Miret, J.J.; Vukovic, S.; Belic, M.R. Engineered surface waves in hyperbolic metamaterials. Opt. Express 2013, 21, 19113-19127. [CrossRef] [PubMed]

2. Jacob, Z.; Narimanov, E.E. Optical hyperspace for plasmons: Dyakonov states in metamaterials. Appl. Phys. Lett. 2008, 93, 221109. [CrossRef]

3. Maier, S.A. Plasmonics. In Fundamentals and Applications; Springer: Heidelberg, Germany, 2007.

4. Gric, T. Surface-plasmon-polaritons at the interface of nanostructured metamaterials. Prog. Electromagn. Rec. 2016, 46, 165-172. [CrossRef]

5. Gric, T.; Wartak, M.S.; Cada, M.; Wood, J.J.; Hess, O.; Pistora, J. Spoof plasmons in corrugated semiconductors. J. Electromagn. Waves Appl. 2015, 29, 1899-1907. [CrossRef]

6. Gric, T. Spoof plasmons in corrugated transparent conducting oxides. J. Electromagn. Waves Appl. 2016, 30, 721-727. [CrossRef]

7. MacDonald, K.F.Z.; Samson, L.; Stockman, M.I.; Zheludev, N.I. Ultrafast active plasmonics. Nat. Photonics 2009, 3, 55-58. [CrossRef]

8. Atwater, H.A.; Polman, A. Plasmonics for improved photovoltaic devices. Nat. Mater. 2010, 9, $205-213$. [CrossRef] [PubMed]

9. Kabashin, A.; Evans, P.; Pastkovsky, S.; Hendren, W.; Wurtz, G.; Atkinson, R.; Pollard, R.; Podolskiy, V.; Zayats, A. Plasmonic nanorod metamaterials for biosensing. Nat. Mater. 2009, 8, 867-871. [CrossRef] [PubMed]

10. Park, Y.; Kim, S.; Choi, J.; Lee, D.-H.; Kim, Y.-J.; Kling, M.F.; Stockman, M.I.; Kim, S.-W. Plasmonic generation of ultrashort extreme-ultraviolet light pulses. Nat. Photonics 2011, 5, 677-681. [CrossRef]

11. Barnes, W.; Dereux, A.; Ebbesen, T. Surface plasmon subwavelength optics. Nature 2003, 424, 824-830. [CrossRef] [PubMed]

12. Shalaev, V.M. Optical negative-index metamaterials. Nat. Photonics 2007, 1, 41-48. [CrossRef]

13. Kim, S.; Jin, J.; Kim, Y.-J.; Park, I.-Y.; Kim, Y.; Kim, S.-W. High-harmonic generation by resonant plasmon field enhancement. Nature 2008, 453, 757-760. [CrossRef] [PubMed]

14. Genevet, P.; Tetienne, J.-P.; Gatzogiannis, E.; Kats, R.M.A.; Scully, M.O.; Capasso, F. Large enhancement of nonlinear optical phenomena by plasmonic nanocavity gratings. Nano Lett. 2010, 10, 4880-4883. [CrossRef] [PubMed]

15. Drachev, V.P.; Podolskiy, V.A.; Kildishev, A.V. Hyperbolic metamaterials: New physics behind a classical problem. Optics. Express 2013, 21, 15048-15064. [CrossRef] [PubMed]

16. Wu, C.; Khanikaev, A.B.; Adato, R.; Arju, N.; Yanik, A.A.; Altug, H.; Shvets, G. Fano-resonant asymmetric metamaterials for ultrasensitive spectroscopy and identification of molecular monolayers. Nat. Mater. 2012, 11, 69-75. [CrossRef] [PubMed]

17. West, P.R.; Kinsey, N.; Ferrera, M.; Kildishev, A.V.; Shalaev, V.M.; Boltasseva, A. Adiabatically tapered hyperbolic metamaterials for dispersion control of high-k waves. Nano Lett. 2015, 15, 498-505. [CrossRef] [PubMed]

18. Chang, Y.-C.; Liu, C.-H.; Liu, C.-H.; Zhang, S.; Marder, S.R.; Narimanov, E.E.; Zhong, Z.; Norris, T.B. Realization of mid-infrared graphene hyperbolic metamaterials. Nat. Commun. 2016, 7, 10568. [CrossRef] [PubMed]

19. Sihvola, A. Electromagnetic Mixing Formulas and Applications; IET: London, UK, 1999.

20. Shekhar, P.; Atkinson, J.; Jacob, Z. Hyperbolic metamaterials: Fundamentals and applications. Nano Converg. 2014, 1, 14. [CrossRef] [PubMed]

21. Gric, T.; Hess, O. Tunable surface waves at the interface separating different graphene-dielectric composite hyperbolic metamaterials. Opt. Express 2017, 25, 11466-11476. [CrossRef] [PubMed]

22. Kretschmann, E.; Raether, H. Radiative decay of non radiative surface plasmons excited by light. Z. Naturforsch. A 1968, 23, 2135-2136. [CrossRef]

23. Hecht, B.; Bielefeld, H.; Novotny, L.; Inouye, Y.; Pohl, D.W. Local excitation, scattering, and interference of surface plasmons. Phys. Rev. Lett. 1996, 77, 1889-1892. [CrossRef] [PubMed] 
24. Tian, J.; Yu, S.; Yan, W.; Qiu, M. Broadband high-efficiency surface-plasmon-polariton coupler with silicon-metal interface. Phys. Lett. 2009, 95, 013504. [CrossRef]

25. Ling, R.T.; Scholler, J.D.; Ufimtsev, P.Ya. The propagation and excitation of surface waves in an absorbing Layer. Prog. Electromagn. Res. 1998, 19, 49-91. [CrossRef]

26. Tumkur, T.U.; Gu, L.; Kitur, J.K.; Narimanov, E.E.; Noginov, M.A. Control of absorption with hyperbolic metamaterials. Appl. Phys. Lett. 2012, 100, 161103. [CrossRef]

27. Sorni, J.A.; Naserpour, M.; Zapata-Rodríguez, C.J.; Miret, J.J. Dyakonov surface waves in lossy metamaterials. Opt. Commun. 2015, 355, 251-255. [CrossRef]

28. Campione, S.; Marquier, F.; Hugonin, J.-P.; Ellis, A.R.; Klem, J.F.; Sinclair, M.B.; Luk, T.S. Directional and monochromatic thermal emitter from epsilon-near-zero conditions in semiconductor hyperbolic metamaterials. Sci. Rep. 2016, 6, 34746. [CrossRef] [PubMed]

29. Vassant, S.; Hugonin, J.-P.; Marquier, F.; Greffet, J.-J. Berreman mode and epsilon near zero mode. Opt. Express 2012, 20, 23971-23977. [CrossRef] [PubMed]

30. Vassant, S.; Archambault, A.; Marquier, F.; Pardo, F.; Gennser, U.; Cavanna, A.; Pelouard, J.; Greffet, J.-J. Epsilon-near-zero mode for active optoelectronic devices. Phys. Rev. Lett. 2012, 109, 237401. [CrossRef] [PubMed]

31. Campione, S.; Brener, I.; Marquier, F. Theory of epsilon-near-zero modes in ultrathin films. Phys. Review B 2015, 91, 121408. [CrossRef]

32. Kliewer, K.; Fuchs, R. Collective electronic motion in a metallic slab. Phys. Rev. 1967, 153, 498-512. [CrossRef]

33. McAlister, A.; Stern, E. Plasma resonance absorption in thin metal films. Phys. Rev. 1963, 132, 1599-1602. [CrossRef]

34. Berreman, D.W. Infrared absorption at longitudinal optic frequency in cubic crystal films. Phys. Rev. 1963, 130, 2193-2198. [CrossRef]

35. Takayama, O.; Artigas, D.; Torner, L. Practical dyakonons. Opt. Lett. 2012, 37, 4311-4313. [CrossRef] [PubMed]

36. Orlov, A.A.; Voroshilov, P.M.; Belov, P.A.; Kivshar, Y.S. Engineered optical nonlocality in nanostructured metamaterials. Phys. Rev. B 2011, 84, 045424. [CrossRef]

37. Dionne, J.A.; Sweatlock, L.A.; Atwater, H.A.; Polman, A. Planar metal plasmon waveguides: Frequency-dependent dispersion, propagation, localization, and loss beyond the free electron model. Phys. Rev. B 2005, 72, 075405. [CrossRef]

38. Naik, G.V.; Shalaev, V.M.; Boltasseva, A. Alternative plasmonic materials: Beyond gold and silver. Adv. Mater. 2013, 25, 3264-3294. [CrossRef] [PubMed]

39. Gric, T.; Hess, O. Controlling hybrid-polarization surface plasmon polaritons in dielectric-transparent conducting oxides metamaterials via their effective properties. J. Appl. Phys. 2017, 122, 193105. [CrossRef]

40. Iorsh, I.; Orlov, A.; Belov, P.; Kivshar, Y. Interface modes in nanostructured metal-dielectric metamaterials. Appl. Phys. Lett. 2011, 99, 151914. [CrossRef]

41. Corson, D.; Lorrain, P. Electromagnetic Fields and Waves; W.H. Freeman: San Francisco, CA, USA, 1962.

(c) 2018 by the authors. Licensee MDPI, Basel, Switzerland. This article is an open access article distributed under the terms and conditions of the Creative Commons Attribution (CC BY) license (http://creativecommons.org/licenses/by/4.0/). 